\title{
Evaluation of efficacy and toxicity of nivolumab combined with or without docetaxel in patients with advanced NSCLC
}

\author{
Yang Wang ${ }^{1}$. Jun Nie ${ }^{1} \cdot$ Ling Dai $^{1} \cdot$ Weiheng Hu${ }^{1} \cdot$ Jie Zhang ${ }^{1}$ Xiaoling Chen ${ }^{1} \cdot{\text { Xiangjuan } \mathrm{Ma}^{1} \cdot \text { Guangming Tian }}^{1}$.

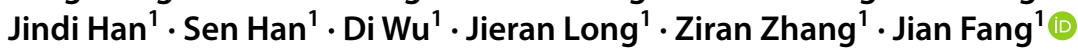

Received: 28 December 2020 / Accepted: 9 May 2021 / Published online: 15 June 2021

(c) The Author(s) 2021

\begin{abstract}
Background The combination of PD-1/PD-L1 inhibitor and chemotherapy has been clinically confirmed to be beneficial as the first-line treatment of patients with advanced NSCLC. This study aimed to assess the effect of nivolumab + docetaxel versus nivolumab monotherapy in patients with NSCLC after the failure of platinum doublet chemotherapy.

Materials and methods The efficacy and toxicity of nivolumab + docetaxel combination therapy versus nivolumab monotherapy were compared in this retrospective study. Primary endpoint of the study was progression-free survival (PFS), and the secondary endpoints were objective response rate (ORR), overall survival (OS), and toxicity.

Results Between November 2017 and December 2019, 77 patients were included in this study, with 58 patients in the nivolumab group and 19 in the nivolumab + docetaxel group. The median follow-up was 18 months, and the PFS was 8 months for patients receiving nivolumab + docetaxel and 2 months for those receiving nivolumab alone $(p=0.001)$, respectively. Nivolumab + docetaxel showed superior OS compared with nivolumab, with the median OS unreached versus 7 months $(p=0.011)$. Among patients without EGFR/ALK variation, compared to nivolumab monotherapy, nivolumab + docetaxel showed better PFS $(p=0.04)$ and OS $(p=0.05)$. There was no significant difference in grade $3-4$ adverse events (AEs) between the two groups $(p=0.253)$.

Conclusions The combination of nivolumab and docetaxel demonstrated a meaningful improvement in progression-free survival and overall survival compared to nivolumab monotherapy, in patients with NSCLC after the failure of platinum doublet chemotherapy, irrespective of EGFR/ALK variation status.
\end{abstract}

Keywords Non-small cell Lung cancer $\cdot$ Nivolumab $\cdot$ Docetaxel $\cdot$ Combination therapy $\cdot$ EGFR $\cdot$ ALK

\section{Introduction}

Lung cancer is the most common malignant tumor and the leading cause of cancer-related deaths worldwide [1]. Among the lung cancer subtypes, non-small cell lung cancer (NSCLC) is most prevalent. Immunotherapy has profoundly changed the treatment modalities and outcomes of advanced NSCLC, as well as significantly improved the tumor response and overall survival (OS). Hence, researchers

Jian Fang

jianfang@bjcancer.org

1 Key Laboratory of Carcinogenesis and Translational Research (Ministry of Education/Beijing), Department of Thoracic Oncology II, Peking University Cancer Hospital \& Institute, Haidian District, 52\# Fucheng Road, Beijing 100142, China continue to explore different combinations of immunotherapy, including dual immunotherapy combination, immunotherapy combined with chemotherapy, immunotherapy combined with targeted therapy, and immunotherapy combined with anti-vascular drugs, in patients with advanced NSCLC. The KEYNOTE-189 and KEYNOTE-407 studies confirmed the survival advantage of pembrolizumab combined with doublet platinum-based chemotherapy as the first-line treatment for NSCLC, compared with doublet platinumbased chemotherapy [2,3]. Similarly, IMPOWER150 and IMPOWER130 studies demonstrated superior survival with atezolizumab combined with chemotherapy in advanced NSCLC [4, 5].

Combination of PD-1/PD-L1 inhibitor and platinum doublet chemotherapy brought significant improvements in survival of patients with advanced NSCLC. Several studies have shown that chemotherapy could increase the infiltration 
of CD8 + T cells and down-regulate the regulatory T cells (Tregs) and convert the non-immunogenic microenvironment to immunogenic, thereby enhancing the activity of immunotherapy $[6,7]$. Preclinical and clinical studies have established the effectiveness and safety of combined PD-1/ PD-L1 inhibitor and chemotherapy as the first-line therapy for advanced NSCLC, which is the preferred treatment option in clinical practice.

As the second-line systemic therapy for NSCLC, nivolumab showed significant improvement in OS versus docetaxel in patients with advanced NSCLC, in both nonsquamous and squamous histology, regardless of PD-L1 expression $[8,9]$. However, nivolumab often had a slow onset of efficacy, with the median time to response of 2.2 months in squamous NSCLC and 2.1 months in nonsquamous NSCLC. In clinical practice, nivolumab was not effective in many patients before deterioration of their general health status or disease progression, thus leading to discontinuation of nivolumab treatment. In addition, nivolumab did not show a significant advantage in progression-free survival (PFS) compared to docetaxel, with a median PFS of 3.5 versus 2.8 months in squamous NSCLC and 2.3 versus 4.3 months in non-squamous NSCLC [10]. Hence, it is important to assess if a similar synergistic activity as in the first-line therapy can be achieved by adding docetaxel to the standard treatment of nivolumab for the second-line therapy of advanced NSCLC. The objective of this retrospective study was to assess the effect of nivolumab + docetaxel combination therapy versus nivolumab monotherapy in patients with NSCLC after the failure of platinum doublet chemotherapy.

\section{Materials and methods}

Patients with stage IIIB to IV NSCLC, who had received nivolumab or nivolumab + docetaxel treatment after the failure of platinum-based chemotherapy at the Department of Thoracic Oncology, Peking University Cancer Hospital and Institute between November 2017 and December 2019, were included in this study. Patients with EGFR/ALK mutationpositive tumors were included if they had progression during or after at least one approved EGFR/ALK inhibitor and platinum-based doublet chemotherapy. Patients who had a poor Eastern Cooperative Oncology Group (ECOG) performance status $(E C O G \geq 3)$ had participated in clinical trials or received more than four lines of systemic therapy were excluded. Patients' data, including clinicopathological characteristics, treatment, and follow-up, were extracted from a prospectively maintained database for NSCLC at the Peking University Cancer Hospital and Institute. All the patients provided informed consent with a signature confirmation before treatment. This study was approved by the Ethics
Committee of the Peking University Cancer Hospital and Institute.

Nivolumab (3 mg/ $\mathrm{kg})$ was given every two weeks in the nivolumab monotherapy group. In the nivolumab + docetaxel group, at the initial phase of nivolumab treatment, all the patients received 4-6 cycles of docetaxel according to the investigator's choice, and docetaxel $\left(75 \mathrm{mg} / \mathrm{m}^{2}\right)$ was given every three weeks. During the cycles combined with docetaxel, nivolumab $(3 \mathrm{mg} / \mathrm{kg}$ ) was delivered every three weeks in order to maintain consistency with the chemotherapy and reduce the number of hospitalizations. After completion of induction chemotherapy, nivolumab $(3 \mathrm{mg} / \mathrm{kg})$ was given every 2 weeks until disease progression or unacceptable toxicity. The PD-L1 expression was tested using PD-L1 IHC 22C3 pharmDx assay.

The primary endpoint of the study was PFS, and the secondary endpoints were objective response rate (ORR), OS, and the incidence and severity of adverse events (AEs). Best overall response (BOR) and disease control rate (DCR) were also evaluated. The PFS was defined as the time from the start of nivolumab or nivolumab + docetaxel treatment until disease progression or death, while OS was defined from the start of nivolumab or nivolumab + docetaxel until death. The tumor responses of patients were assessed by computed tomography (CT) or magnetic resonance imaging (MRI) every 6-8 weeks after starting treatment, according to the Response Evaluation Criteria in Solid Tumor (V1.1). ORR was defined as the proportion of patients achieving complete response (CR) or partial response (PR). DCR was defined as the number of patients achieving CR, PR or stable disease.

Chi-square tests or Fisher's exact tests were used to analyze the statistical significance of categorical variables in the two groups. Independent sample t test was used for analyzing the difference between continuous variables. The difference of ORR and DCR were calculated using two separate chi-square tests (Pearson's chi-square test and Yates's correction for continuity, respectively). PFS and OS were analyzed by Kaplan-Meier survival curves. Differences were determined to be statistically significant if $p$ value $<0.05$. Graphs were plotted by GraphPad Prism software, and data were analyzed by SPSS and GraphPad Prism.

\section{Results}

\section{Patient characteristics}

A total of 77 patients were enrolled in this retrospective study between November 2017 and December 2019, with 58 patients in the nivolumab group and 19 in the nivolumab + docetaxel group. The baseline characteristics (Table 1), including gender, age, histology, stage at diagnosis, ECOG status, EGFR/ALK variation status, PD-L1 
Table 1 Patient baseline characteristics in nivolumab group and nivolumab combined docetaxel group

\begin{tabular}{|c|c|c|c|c|}
\hline Variable & $\begin{array}{l}\text { All patients } n=77 \\
(\%)\end{array}$ & $\begin{array}{l}\text { Nivolumab } n=58 \\
(\%)\end{array}$ & $\begin{array}{l}\text { Nivolumab + Docetaxel } \\
n=19(\%)\end{array}$ & $p$ value \\
\hline Age (years, median) & 62 & 63 & 60 & 0.697 \\
\hline$\geq 65$ & $29(37.7)$ & $23(39.7)$ & $6(31.6)$ & 0.582 \\
\hline Gender, man & $54(70.1)$ & $40(69)$ & $24(73.7)$ & \\
\hline \multicolumn{5}{|l|}{ Histology } \\
\hline Non-squamous & $39(50.6)$ & $28(48.3)$ & $11(57.9)$ & \multirow[t]{2}{*}{0.467} \\
\hline Squamous & $38(49.4)$ & $30(51.7)$ & $8(42.1)$ & \\
\hline \multicolumn{5}{|l|}{ ECOG } \\
\hline $0-1$ & $67(87)$ & $49(84.5)$ & $18(94.7)$ & \multirow[t]{2}{*}{0.436} \\
\hline 2 & $10(13)$ & $9(15.5)$ & $1(5.3)$ & \\
\hline \multicolumn{5}{|l|}{ Stage at diagnosis } \\
\hline IIIb-IIIc & $3(3.9)$ & $3(5.2)$ & 0 & \multirow[t]{2}{*}{0.571} \\
\hline IV & $74(96.1)$ & $55(94.8)$ & $19(100 \%)$ & \\
\hline \multicolumn{5}{|l|}{ PD-L1 expression } \\
\hline Positive & $22(28.6)$ & $16(27.6)$ & $6(31.6)$ & \multirow[t]{3}{*}{0.899} \\
\hline Negative & $15(19.5)$ & $11(19)$ & $4(21.1)$ & \\
\hline Undetected & $40(51.9)$ & $31(53.3)$ & $9(47.4)$ & \\
\hline \multicolumn{5}{|l|}{ Oncotarget variation } \\
\hline EGFR/ALK positive & $9(11.7)$ & $7(12.1)$ & $2(10.5)$ & 0.856 \\
\hline \multicolumn{5}{|l|}{ Therapeutic lines } \\
\hline Second line & $42(54.5)$ & $25(43.1)$ & $17(89.5)$ & \multirow[t]{3}{*}{0.002} \\
\hline Third line & $27(35.1)$ & $25(43.1)$ & $2(10.5)$ & \\
\hline Fourth line & $8(10.4)$ & $8(13.8)$ & 0 & \\
\hline \multicolumn{5}{|l|}{ Brain metastasis } \\
\hline Yes & $54(70.1)$ & $41(70.7)$ & $13(68.4)$ & \multirow[t]{2}{*}{0.851} \\
\hline No & $23(29.9)$ & $17(29.3)$ & $6(31.6)$ & \\
\hline
\end{tabular}

expression, and brain metastasis, were generally similar between the two groups, although the therapeutic lines when receiving nivolumab monotherapy or combination therapy had a significant difference between the two groups $(p=0.02)$. A total of nine patients $(9 / 77,11.7 \%)$ with EGFR $(7 / 77,9.1 \%)$ or ALK variations $(2 / 77,2.6 \%)$ were included in this study (seven patients with EGFR variation and two with ALK variation). All nine patients with sensitizing EGFR or ALK mutations received corresponding targeted therapy before platinum-based chemotherapy. Only 37 of 77 patients $(46.6 \%)$ underwent the PD-L1 expression test, and the proportion of PD-L1-positive patients was similar between the two groups.

The median number of cycles of nivolumab administered was 5 (range 1-37) in the nivolumab group and 6 (range 2-18) in the nivolumab + docetaxel group. The median follow-up in the nivolumab group was 19 months (range 1-42), and 8 of 58 patients (13.7\%) were still receiving the nivolumab treatment. The median follow-up in the nivolumab + docetaxel group was 11 months (range $1-22)$, and 8 of 19 patients $(42.1 \%)$ were still receiving the nivolumab treatment. Meanwhile, 26 of 58 patients $(44.8 \%)$ in the nivolumab group and 10 of 18 patients $(55.6 \%)$ in the nivolumab + docetaxel group had received subsequent therapy, and the most common therapy was anlotinib (a small molecule target-multiple tyrosine kinase inhibitor, which was approved for use in third-line therapy of NSCLC in China). Nineteen patients $(52.8 \%)$ received anlotinib as the subsequent therapy. Other subsequent therapy included pemetrexed (5 patients, 13.9\%), docetaxel (4 patients, 11.1\%), S-1 (4 patients, 11.1\%), and nab-paclitaxel (4 patients, $11.1 \%)$.

\section{Efficacy}

Best change in target lesions from baseline for all participants is listed in Fig. 1. The ORR was $15.5 \%(95 \%$ CI $6.2-24.8 \%)$ vs. $21.1 \%$ (95\% CI $2.7-39.4 \%)(p=0.576)$ in the nivolumab group vs. the nivolumab + docetaxel group, respectively. No patient achieved CR. The DCR was $48.3 \%$ (95\% CI 35.4-61.1\%) in the nivolumab group and $94.7 \%$ (95\% CI 84.7-100\%) in the nivolumab + docetaxel group $(p<0.001)$. Among patients with EGFR-wild-type and ALK negative, DCR was $48.3 \%$ (95\% CI 35.4-61.1\%) in the nivolumab group and $94.7 \%$ (95\% CI 84.7-100\%) in the nivolumab + docetaxel group $(p<0.001)$, but ORR 
Fig. 1 Best change in target lesions from baseline for all population. ORR, overall response rate; DCR, disease control rate

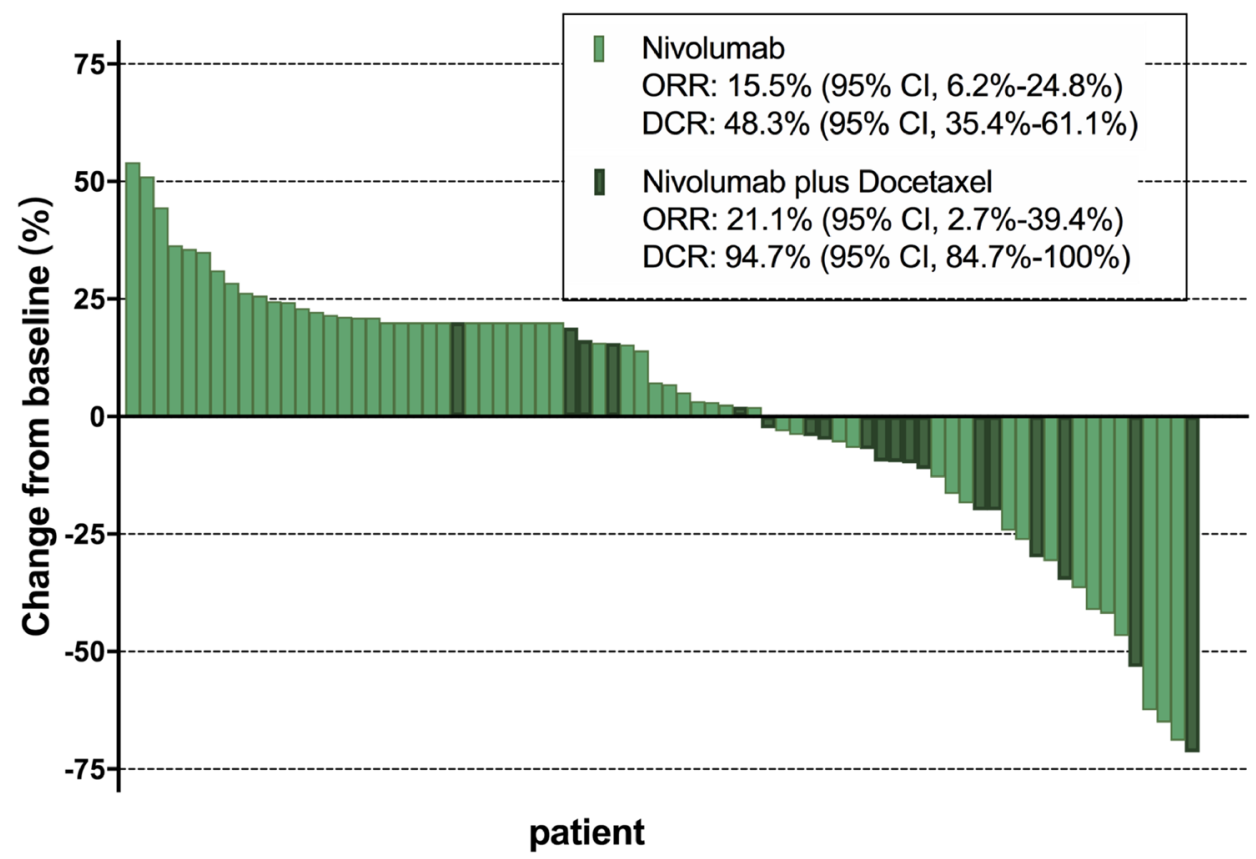

ORR: overall response rate, DCR: disease control rate. did not show statistical difference between the two groups $(p=0.477)$.

The nivolumab + docetaxel group had a significantly prolonged PFS compared with nivolumab alone (8 months; 95\% CI 1.2-14.7; vs. 2 months; 95\% CI 1.6-2.4; $p=0.001$ ) (Fig. 2A). Nivolumab + docetaxel also showed superior OS compared with nivolumab alone (Fig. 2B), with the median OS unreached in the nivolumab + docetaxel group versus 7 months (95\% CI 4.4-9.6) $(p=0.011)$ in the nivolumab group. Improved PFS and OS with nivolumab + docetaxel were also observed in the EGFR/ALK-negative subgroup. Among patients without EGFR/ALK variation, median PFS was 7 months (95\% CI 2.4-11.6) with nivolumab + docetaxel and 3 months (95\% CI 2.6-3.4) with nivolumab alone $(p=0.04)$; median OS was not reached with nivolumab + docetaxel and 8 months (95\% CI 5.0-11.0) with nivolumab alone $(p=0.05)$. For patients with brain metastasis, the nivolumab + docetaxel group had a significantly prolonged PFS compared with nivolumab alone (unreached; vs. 3 months; $p=0.03$ ), but the OS did not show survival benefits $(p=0.187)$.

Subgroup analysis showed nivolumab + docetaxel brought benefits in most pre-specified subgroups.

Since the therapeutic lines in the two groups were imbalanced, we analyzed the PFS and OS in the second-line therapy subgroup. For patients receiving the second-line therapy, median PFS was 8 months (95\% CI 1.2-14.8) with nivolumab + docetaxel and 2 months (95\% CI 1.5-2.6) with nivolumab alone ( $p=0.01$, Fig. $3 \mathrm{~A})$; median OS was not reached with nivolumab + docetaxel and 8 months $(95 \%$ CI 2.7-13.2) with nivolumab alone ( $p=0.04$, Fig. 3B). Nivolumab + docetaxel showed a significant advantage for PFS and OS compared with nivolumab monotherapy in second-line therapy. We also compared the PFS and OS in patients with squamous histology vs non-squamous histology. The PFS $(p=0.112)$ and OS $(p=0.29)$ had no significant difference between patients with squamous and nonsquamous histology.

\section{Toxicity}

Any-grade adverse events (AEs) were more frequent with nivolumab + docetaxel $(14 / 19,73.7 \%)$ than nivolumab alone $(24 / 58,41.4 \%)(p=0.015)$, but grade 3-4 AEs did not display a significant difference between the two groups (10.5\% vs. $3.4 \%, p=0.253$ ). All-causality, any-grade AEs in the whole cohort are shown in Fig. 4. The most frequent treatment-related AEs in the nivolumab + docetaxel arm included fatigue $(21.1 \%)$, nausea $(15.8 \%)$, leukopenia and neutropenia (15.8\%), pneumonitis (15.8\%), rash (10.5\%), fever (10.5\%), and decreased appetite (5.3\%). In the nivolumab monotherapy group, the most frequent treatment-related AEs included fatigue $(12.1 \%)$, rash $(12.1 \%)$, decreased appetite $(5.2 \%)$, pneumonitis (3.4\%), and nausea (1.7\%). Of these, the frequencies of nausea, leukopenia and neutropenia were higher in the nivolumab + docetaxel group than in the nivolumab group. Compared to nivolumab alone, nivolumab + docetaxel might increase the incidence of pneumonitis (15.8\% 
Fig. 2 Kaplan-Meier curves for progression-free survival (A) and overall survival $(\mathbf{B})$ for all patients

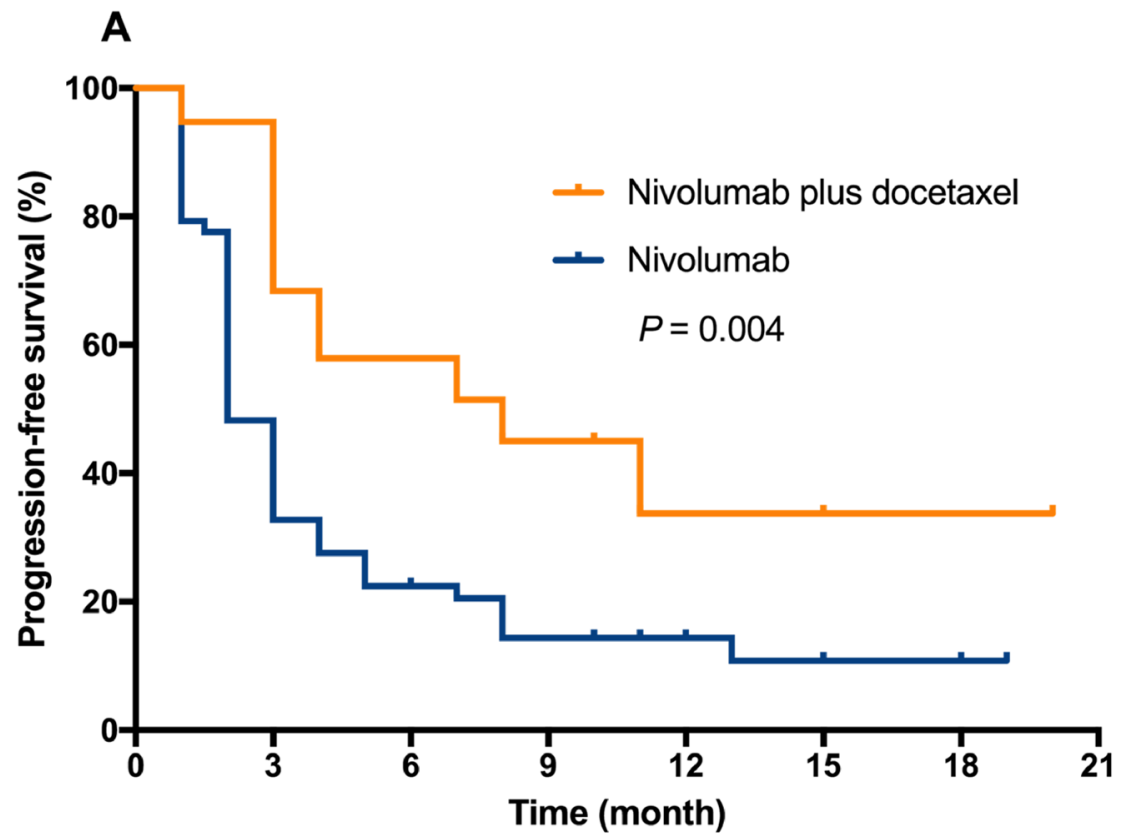

No. at risk

\begin{tabular}{|c|c|c|c|c|c|c|}
\hline $\begin{array}{l}\text { Nivolumab plus } 19 \\
\text { chemotherapy }\end{array}$ & 17 & 7 & 5 & 1 & 1 & 1 \\
\hline Nivolumab $\quad 58$ & 27 & 12 & 6 & 4 & 2 & 1 \\
\hline
\end{tabular}

B

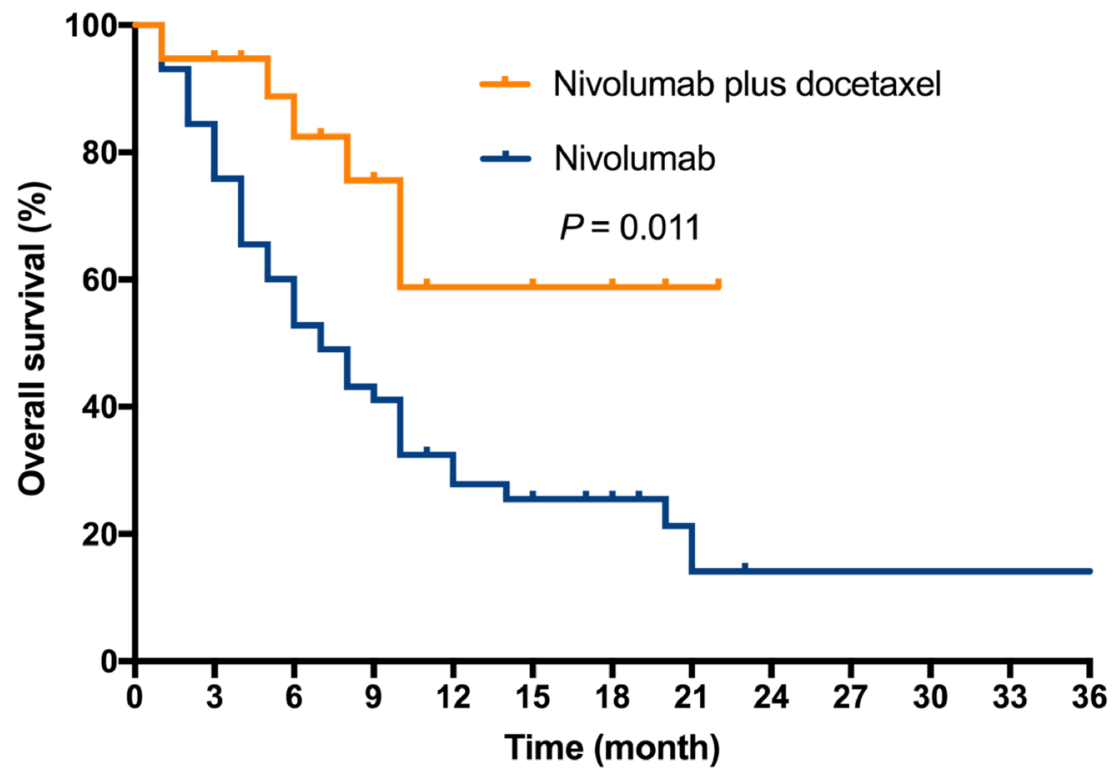

No. at risk

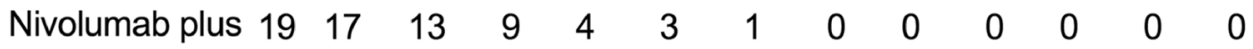
chemotherapy

$\begin{array}{llllllllllllll}\text { Nivolumab } & 58 & 48 & 32 & 20 & 13 & 10 & 8 & 2 & 1 & 1 & 1 & 1 & 1\end{array}$

vs. $3.4 \%)$, though not statistically significant $(p=0.174)$. There was no grade 3-4 pneumonitis in the entire cohort.

The uncommon AEs in the nivolumab + docetaxel group included one patient with grade 1 transaminase elevation. In the nivolumab monotherapy group, one patient developed hypothyroidism, one had asymptomatic amylase elevation, while two patients developed venous thrombosis, which could also be caused by lung cancer, and thus the relation 
Fig. 3 Kaplan-Meier curves for progression-free survival (A) and overall survival (B) for patients on second-line therapy

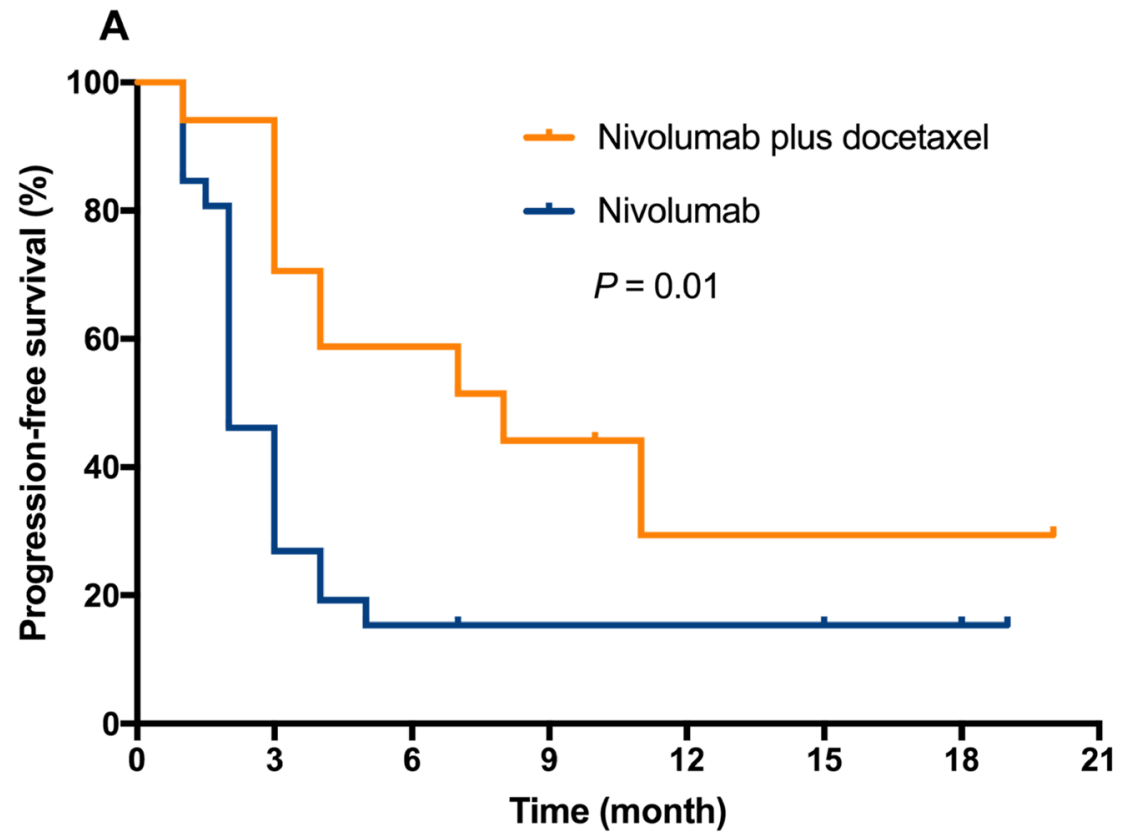

No. at risk

$\begin{array}{llllllll}\begin{array}{l}\text { Nivolumab plus } \\ \text { chemotherapy }\end{array} & 15 & 7 & 4 & 1 & 1 & 1 & 0 \\ \begin{array}{l}\text { Nivolumab } \\ \text { Nivoluma }\end{array} & 11 & 3 & 3 & 3 & 2 & 1 & 0\end{array}$

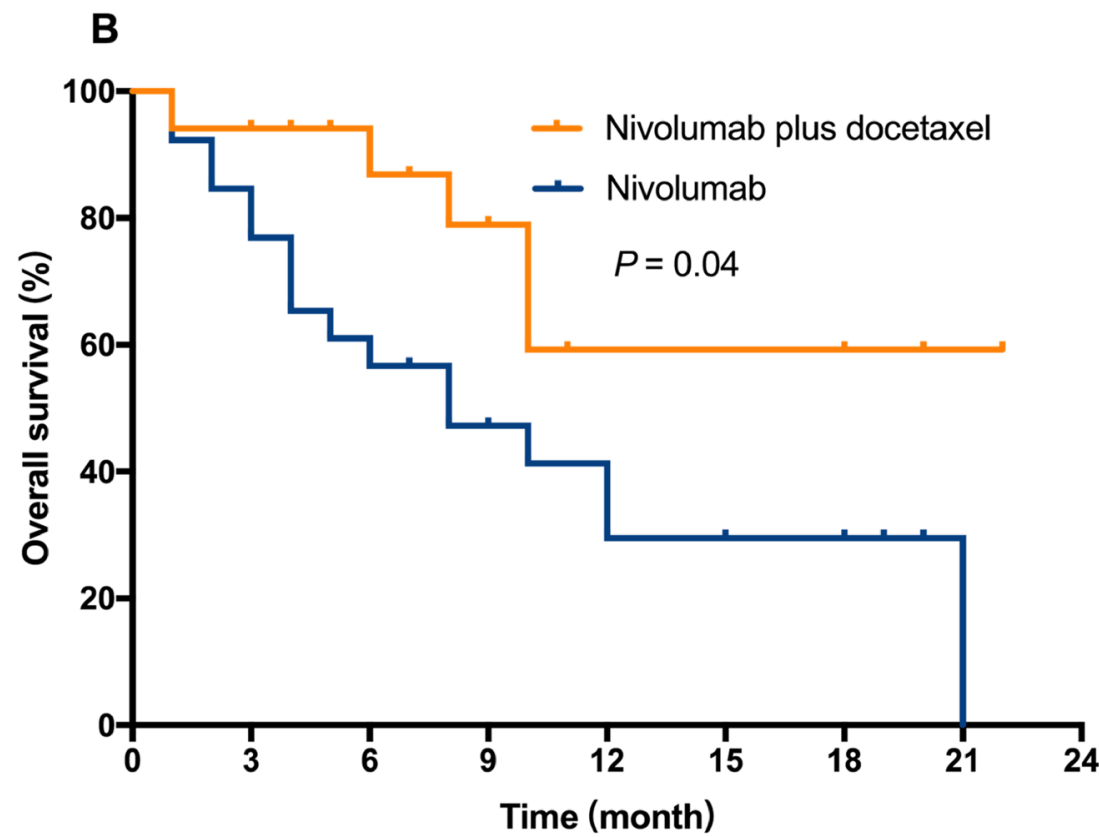

No. at risk

$\begin{array}{lllllllll}\begin{array}{l}\text { Nivolumab plus } \\ \text { chemotherapy }\end{array} & 15 & 12 & 8 & 2 & 2 & 2 & 1 & 0 \\ \begin{array}{l}\text { Nivolumab } \\ \text { Nivoluma }\end{array} & 21 & 13 & 8 & 6 & 4 & 3 & 0 & 0\end{array}$

to nivolumab treatment was uncertain. One patient in each i.e., grade 2 pneumonia. There were no grade 4 toxicities and group discontinued treatment due to treatment-related AEs, treatment-related deaths in the two groups. 
Fig. 4 Treatment-related adverse events in patients treated with nivolumab $(\mathbf{A})$ or nivolumab plus docetaxel (B)
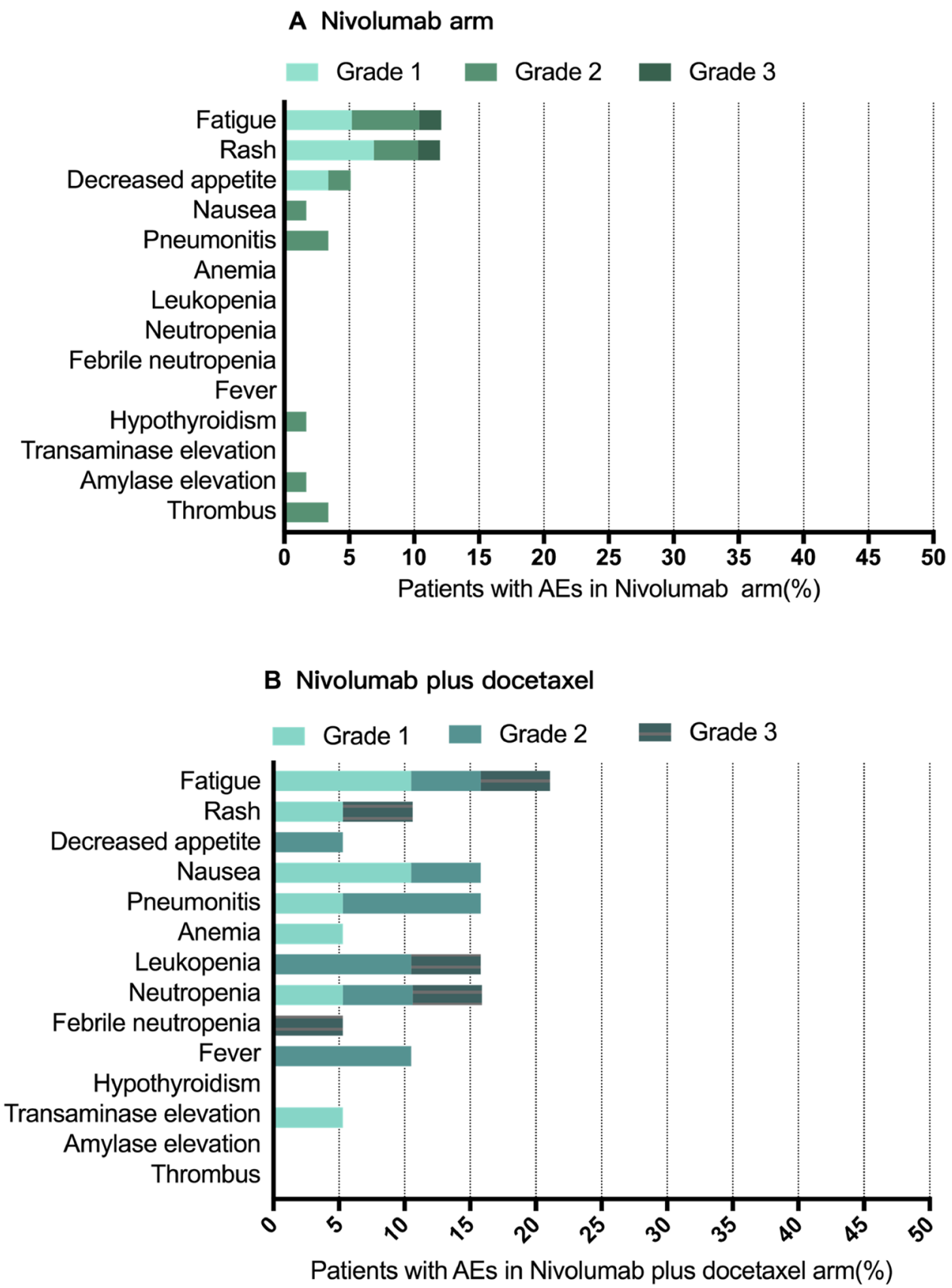

\section{Discussion}

According to the results of the CheckMate 017 and CheckMate 057 studies [8, 9], nivolumab treatment has become the new standard second-line or above treatment for NSCLC patients. In two phase III clinical studies, nivolumab showed a longer OS and fewer AEs than docetaxel in squamous and non-squamous NSCLC. The analysis of long-term results confirmed that nivolumab had significant clinical efficacy compared with docetaxel $[10,11]$. The ORR of nivolumab was $19 \%$ for adenocarcinoma and $20 \%$ for squamous cell carcinoma, while ORR of docetaxel was $12 \%$. Nivolumab showed lasting disease remission, but only in limited patients. The median treatment duration in the pooled CheckMate 017/057 studies for nivolumab and docetaxel was 2.8 (range 0-51.8) months and 2.1 (range 0-20.0) months, respectively, which suggested that a considerable number of patients could not benefit from the second-line nivolumab therapy. Therefore, as in the first-line therapy of NSCLC, perhaps more patients could benefit from the combination of nivolumab and chemotherapy. Recently, a phase II study compared the efficacy and safety of pembrolizumab + docetaxel with docetaxel monotherapy in patients with advanced NSCLC who were previously treated with 
platinum-based chemotherapy [12]. The results showed that the combination of pembrolizumab + docetaxel was safe, and substantially improved ORR and PFS compared with docetaxel. However, no clinical study has directly compared immunotherapy with immunotherapy + chemotherapy in the second-line treatment of NSCLC. Only a small retrospective study indicated that PD-1/PD-L1 inhibitor + nab-paclitaxel showed significantly longer OS and higher response than PD-1/PD-L1 inhibitor monotherapy in second-line and above therapy for NSCLC [13]. However, the majority of patients in the study received third-line and above therapy (65\%), and different types of PD-1/PD-L1 inhibitors were included, which were unreported.

To the best of our knowledge, this is the first study to report that the combination of nivolumab and docetaxel was effective and safe in patients with NSCLC progressing after platinum-based chemotherapy, and had an advantage on PFS and OS compared to the standard nivolumab monotherapy. The median PFS of nivolumab monotherapy in this study was 2 months, which was similar to the reported data in clinical studies and daily practice $[8,9,14-16]$, and the median PFS of nivolumab combined with docetaxel was significantly longer at 8 months. Similarly, compared with nivolumab alone, the OS of nivolumab + docetaxel was significantly longer (unreached vs. 7 months, $p=0.011$ ). The ORR of nivolumab in this study (15.5\%) was slightly lower than previous reports (17.6-22.3\%) [17-19], perhaps because in this study almost $57 \%$ of the patients were receiving nivolumab as a third or fourth line of therapy. Furthermore, it caused the median OS of nivolumab in this study was lower than it was reported in Checkmate 017 and Checkmate $057[8,9]$. Another reason could be that, in our study, patients with performance status ECOG score comprised $13 \%$, and patients with brain metastasis comprised $29.9 \%$. Although the response rate of the two groups did not show statistical differences $(21.1 \%$ vs. $15.5 \%, p=0.576)$, the DCR of the combination group of nivolumab and docetaxel was significantly better than that of the nivolumab group $(94.7 \%$ vs. $48.3 \%, p<0.001)$. This showed that the combination therapy could enable more patients to receive immunotherapy of nivolumab for a longer time.

The therapeutic lines were imbalanced in the two treatment groups, and the nivolumab group had more patients receiving third-line or fourth-line therapy, suggesting that any adverse biases between the two groups should be ruled out. Thus, we analyzed the PFS and OS in secondline therapy between the two groups. Results showed that in the second-line therapy subgroup, addition of docetaxel to nivolumab demonstrated higher PFS (8 months, $95 \%$ CI $1.2-14.8$ vs. 2 months, $95 \%$ CI $1.5-2.6 ; p=0.01)$ and OS (not reached vs. 8 months, 95\% CI 2.7-13.2; $p=0.04$ ), which indicated that nivolumab + docetaxel was generally applicable in second-line and follow-up treatment.
In this study, in the whole population as well as in the patients without EGFR/ALK variation, nivolumab + docetaxel demonstrated higher PFS and OS, compared with nivolumab monotherapy. Previous studies showed that immune checkpoint inhibitor (ICI) monotherapy had a poor treatment efficacy in advanced NSCLC patients with sensitizing EGFR mutations. In the subgroup analysis, nivolumab, pembrolizumab and atezolizumab showed comparable PFS and OS as docetaxel [9, 20, 21]. However, the combination of ICIs and chemotherapy may bring survival advantage to patients with EGFR mutations. The Impower150 study first reported that the combination of ICI and chemotherapy displayed higher OS versus chemotherapy alone [22]. Similarly, a phase II trial compared pembrolizumab + docetaxel with docetaxel monotherapy in patients with failed platinum-based chemotherapy, and found that the PFS benefit was also maintained in patients with EGFR variations [12]. Currently, several clinical trials (NCT02864251, NCT03515837) are exploring the beneficial effects of immunotherapy in EGFR-mutant populations.

The safety profile of nivolumab + docetaxel was in accordance with the reported AEs related to nivolumab or docetaxel, and no new AEs were observed. There was no significant difference between the two groups in the proportion of grade 3-4 AEs (10.5\% in the nivolumab + docetaxel group vs. $3.4 \%$ in the nivolumab group, $p=0.253$ ). No fatal AEs occurred in the two groups. Notably, the incidence of pneumonitis in the nivolumab + docetaxel group was higher than in the nivolumab monotherapy group (15.8\% vs. $3.4 \%)$, although not statistically significant. The possible cause of the difference is that pneumonia is a common AE of docetaxel chemotherapy. In a large-scale, real-world analysis of patients with NSCLC who received docetaxel as second-line therapy, atypical pneumonia was the third most common $\mathrm{AE}$, recorded in $18 \%$ of patients, and $10-12 \%$ of patients needed emergency visits or treatment [23]. The frequency of pneumonitis previously observed with nivolumab monotherapy ranged from 1 to $10.8 \%$ in clinical trials and realworld studies [9, 10, 14, 15, 24, 25]. The age, ECOG status, and pre-existing pulmonary conditions of patients included in the studies affected the frequency of treatment-related pneumonitis. Given the low number of cases analyzed in the nivolumab + docetaxel group, the incidence of pneumonia with combined treatment needs further confirmation.

This study had several limitations. First, the non-randomized and retrospective study design led to unavoidable survival and selection biases. We are currently recruiting patients for a prospective, randomized controlled trial to compare the efficacy and safety of nivolumab monotherapy with nivolumab + docetaxel in advanced NSCLC patients with failed platinum-based chemotherapy. Second, the status of PD-L1 expression was undetected in most patients so we could not perform subgroup analysis with different 
PD-L1 expression levels. It is not necessary to test PD-L1 expression before nivolumab treatment. Third, the number of patients in this study was small, especially in the nivolumab combined chemotherapy group.

In summary, this retrospective study showed that the combination of nivolumab and docetaxel demonstrated a meaningful improvement in progression-free survival and overall survival, in patients with NSCLC after the failure of platinum doublet chemotherapy, irrespective of EGFR/ ALK variation status. This suggested that the combination of immunotherapy and chemotherapy could be a potential treatment choice in NSCLC second-line therapy. As the nivolumab often had a slow onset of efficacy in realworld practice, and the hyperprogressions made patients had little opportunity to receive the third or fourth line of therapies because of bad general conditions. The hyperprogression has an incidence of $6-43 \%$ in ICIs monotherapy and has not been reported in combination therapy of ICIs and chemotherapy [26]. The attempts to combine the nivolumab and chemotherapy in second-line therapy in NSCLC patients are clinically significant. Prospective randomized controlled study is needed.

Acknowledgements The authors would like thank the patients and their families for providing information and agreeing to the analysis.

Authors' contributions YW and JF planned and designed the study. YW collected and analyzed the data and wrote the article. JN, LD, WH, $\mathrm{XC}, \mathrm{XM}$, and GT collected data. JH, SH, and DW helped to conduct the literature review. JL, ZZ, and JZ helped to analyze the data. All authors read and approved the final version.

Funding The study was supported by Chinese Society of Clinical Oncology-Bristol-Myers Squibb Cancer Immunology Research Fund (Y-BMS2019-010).

Availability of data and material All data generated or analyzed during this study are included in this published article.

\section{Code availability None.}

\section{Declarations}

Conflict of interest None of the authors have actual or potential conflicts of interest, including any financial, personal or other relationships with other people or organizations that could inappropriately influence this article, to declare.

Consent to participate All participants provide their written informed consent before their treatment of nivolumab or nivolumab + docetaxel.

Consent for publication All participants provide their written informed consent before their treatment of nivolumab or nivolumab + docetaxel.

Ethics approval The authors state that they have obtained appropriate institutional review board approval or have followed the principles outlined in the Declaration of Helsinki for all human or animal experimental investigations.
Open Access This article is licensed under a Creative Commons Attribution 4.0 International License, which permits use, sharing, adaptation, distribution and reproduction in any medium or format, as long as you give appropriate credit to the original author(s) and the source, provide a link to the Creative Commons licence, and indicate if changes were made. The images or other third party material in this article are included in the article's Creative Commons licence, unless indicated otherwise in a credit line to the material. If material is not included in the article's Creative Commons licence and your intended use is not permitted by statutory regulation or exceeds the permitted use, you will need to obtain permission directly from the copyright holder. To view a copy of this licence, visit http://creativecommons.org/licenses/by/4.0/.

\section{References}

1. Bray F, Ferlay J, Soerjomataram I et al (2018) Global cancer statistics 2018: GLOBOCAN estimates of incidence and mortality worldwide for 36 cancers in 185 countries. CA Cancer J Clin 68:394-424. 2018/09/13. https://doi.org/10.3322/caac.21492

2. Gandhi L, Rodríguez-Abreu D, Gadgeel S et al (2018) Pembrolizumab plus chemotherapy in metastatic non-small-cell lung cancer. New Engl J Med 378:2078-2092. 2018/04/17. https://doi.org/ 10.1056/NEJMoa1801005

3. Paz-Ares L, Luft A, Vicente D et al (2018) Pembrolizumab plus chemotherapy for squamous non-small-cell lung cancer. New Engl J Med 379:2040-2051. 2018/10/04. https://doi.org/10.1056/ NEJMoa1810865

4. Socinski MA, Jotte RM, Cappuzzo F et al (2018) Atezolizumab for first-line treatment of metastatic nonsquamous NSCLC. New Engl J Med 378:2288-2301. 2018/06/05. https://doi.org/10.1056/ NEJMoa1716948

5. West H, McCleod M, Hussein M et al (2019) Atezolizumab in combination with carboplatin plus nab-paclitaxel chemotherapy compared with chemotherapy alone as first-line treatment for metastatic non-squamous non-small-cell lung cancer (IMpower130): a multicentre, randomised, open-label, phase 3 trial. Lancet Oncol 20:924-937. 2019/05/28. https://doi.org/10.1016/s1470-2045(19) 30167-6

6. Tseng CW, Hung CF, Alvarez RD et al (2008) Pretreatment with cisplatin enhances E7-specific CD8+ T-Cell-mediated antitumor immunity induced by DNA vaccination. Clin Cancer Res Off J Am Assoc Cancer Res 14:3185-3192. 2008/05/17. https://doi. org/10.1158/1078-0432.Ccr-08-0037

7. Pfirschke C, Engblom C, Rickelt $S$ et al (2016) Immunogenic chemotherapy sensitizes tumors to checkpoint blockade therapy. Immunity 44:343-354. 2016/02/14. https://doi.org/10.1016/j. immuni.2015.11.024

8. Brahmer J, Reckamp KL, Baas P et al (2015) Nivolumab versus Docetaxel in advanced squamous-cell non-small-cell lung cancer. New Engl J Med 373:123-135. 2015/06/02. https://doi.org/10. 1056/NEJMoa1504627

9. Borghaei H, Paz-Ares L, Horn L et al (2015) Nivolumab versus Docetaxel in advanced nonsquamous non-small-cell lung cancer. New Engl J Med 373:1627-1639. 2015/09/29. https://doi.org/10. 1056/NEJMoa1507643

10. Horn L, Spigel DR, Vokes EE et al (2017) Nivolumab versus Docetaxel in previously treated patients with advanced non-smallcell lung cancer: two-year outcomes from two randomized, openlabel, phase III trials (CheckMate 017 and CheckMate 057). J Clin Oncol 35:3924-3933. 2017/10/13. https://doi.org/10.1200/JCO. 2017.74.3062

11. Vokes EE, Ready N, Felip E et al (2018) Nivolumab versus docetaxel in previously treated advanced non-small-cell lung cancer 
(CheckMate 017 and CheckMate 057): 3-year update and outcomes in patients with liver metastases. Ann Oncol Off J Eur Soc Med Oncol 29:959-965. 2018/02/07. https://doi.org/10.1093/ annonc/mdy041

12. Arrieta O, Barrón F, Ramírez-Tirado LA et al (2020) Efficacy and safety of Pembrolizumab plus Docetaxel versus Docetaxel alone in patients with previously treated advanced non-small cell lung cancer: the PROLUNG phase 2 randomized clinical trial. JAMA Oncol 6:1-9. 2020/04/10. https://doi.org/10.1001/jamao ncol.2020.0409

13. Zhang F, Huang D, Zhao L et al (2020) Efficacy and safety of PD-1/PD-L1 inhibitors plus nab-paclitaxel for patients with nonsmall cell lung cancer who have progressed after platinum-based chemotherapy. Ther Adv Med Oncol 12:1758835920936882. 2020/07/17. https://doi.org/10.1177/1758835920936882

14. Morita R, Okishio K, Shimizu J et al (2020) Real-world effectiveness and safety of nivolumab in patients with non-small cell lung cancer: a multicenter retrospective observational study in Japan. Lung Cancer (Amsterdam, Netherlands) 140:8-18. 2019/12/16. https://doi.org/10.1016/j.lungcan.2019.11.014

15. Grossi F, Genova C, Crinò L et al (2019) Real-life results from the overall population and key subgroups within the Italian cohort of nivolumab expanded access program in non-squamous nonsmall cell lung cancer. Eur J Cancer (Oxford, England: 1990) 123:72-80. 2019/11/02. https://doi.org/10.1016/j.ejca.2019.09. 011

16. Dudnik E, Moskovitz M, Daher S et al (2018) Effectiveness and safety of nivolumab in advanced non-small cell lung cancer: the real-life data. Lung Cancer (Amsterdam, Netherlands) 126:217223. 2017/12/20. https://doi.org/10.1016/j.lungcan.2017.11.015

17. Schouten RD, Muller M, de Gooijer CJ et al (2018) Real life experience with nivolumab for the treatment of non-small cell lung carcinoma: data from the expanded access program and routine clinical care in a tertiary cancer centre-The Netherlands Cancer Institute. Lung cancer (Amsterdam, Netherlands) 126:210-216. 2017/11/29. https://doi.org/10.1016/j.lungcan.2017.11.012

18. Merino Almazán M, Duarte Pérez JM, Marín Pozo JF et al (2019) A multicentre observational study of the effectiveness, safety and economic impact of nivolumab on non-small-cell lung cancer in real clinical practice. Int J Clin Pharm 41:272-279. 2018/12/24. https://doi.org/10.1007/s11096-018-0772-z

19. Figueiredo A, Almeida MA, Almodovar MT et al (2020) Realworld data from the Portuguese Nivolumab Expanded Access Program (EAP) in previously treated Non Small Cell Lung Cancer
(NSCLC). Pulmonology 26:10-17. 2019/10/22. https://doi.org/ 10.1016/j.pulmoe.2019.06.001

20. Herbst RS, Baas P, Kim DW et al (2016) Pembrolizumab versus Docetaxel for previously treated, PD-L1-positive, advanced nonsmall-cell lung cancer (KEYNOTE-010): a randomised controlled trial. Lancet (London, England) 387:1540-1550. 2015/12/30. https://doi.org/10.1016/s0140-6736(15)01281-7

21. Rittmeyer A, Barlesi F, Waterkamp D et al (2017) Atezolizumab versus Docetaxel in patients with previously treated non-smallcell lung cancer (OAK): a phase 3, open-label, multicentre randomised controlled trial. Lancet (London, England) 389: 255-265. 2016/12/17. https://doi.org/10.1016/s0140-6736(16)32517-x

22. Reck M, Mok TSK, Nishio M et al (2019) Atezolizumab plus bevacizumab and chemotherapy in non-small-cell lung cancer (IMpower150): key subgroup analyses of patients with EGFR mutations or baseline liver metastases in a randomised, open-label phase 3 trial. Lancet Respir Med 7:387-401. 2019/03/30. https:// doi.org/10.1016/s2213-2600(19)30084-0

23. Arunachalam A, Li H, Bittoni MA et al (2018) Real-world treatment patterns, overall survival, and occurrence and costs of adverse events associated with second-line therapies for medicare patients with advanced non-small-cell lung cancer. Clin Lung Cancer 19:e783-e799. 2018/07/10. https://doi.org/10.1016/j.cllc. 2018.05.016

24. Martin C, Lupinacci L, Perazzo F et al (2020) Efficacy and safety of nivolumab in previously treated patients with non-small-cell lung cancer: real world experience in Argentina. Clin Lung Cancer 2020/03/28. https://doi.org/10.1016/j.cllc.2020.02.014

25. Okishio K, Morita R, Shimizu J et al (2020) Nivolumab treatment of elderly Japanese patients with non-small cell lung cancer: subanalysis of a real-world retrospective observational study (CA2099CR). ESMO Open 5 2020/07/22. https://doi.org/10.1136/esmoo pen-2019-000656

26. Park HJ, Kim KW, Won SE et al (2021) Definition, incidence, and challenges for assessment of hyperprogressive disease during cancer treatment with immune checkpoint inhibitors: a systematic review and meta-analysis. JAMA Netw Open 4:e211136. 2021/03/25. https://doi.org/10.1001/jamanetworkopen.2021.1136

Publisher's Note Springer Nature remains neutral with regard to jurisdictional claims in published maps and institutional affiliations. 